\title{
Soft Gluon Resummation for the Associated Single Top and Higgs Production at the LHC
}

\author{
Anna Kulesza, ${ }^{a}$ Laura Moreno Valero ${ }^{a, *}$ and Vincent Theeuwes ${ }^{b}$ \\ a Institute for Theoretical Physics, WWU Münster, \\ D-48149 Münster, Germany \\ ${ }^{b}$ Institute for Theoretical Physics, Georg-August-Univesity Göttingen, \\ Friedrich-Hund-Platz 1, 37077 Göttingen, Germany \\ E-mail: anna.kulesza@uni-muenster.de, lmore_02@uni-muenster.de, \\ vtheeuwe@gmail.com
}

We study the effects of soft gluon resummation on the $s$-channel $p p \rightarrow H t j$ cross section at the LHC. We perform resummation in the invariant mass threshold at next-to-leading-logarithmic accuracy (NLL), additionally including $O\left(\alpha_{s}\right)$ non-logarithmic contributions. The numerical results presented show an improvement of the scale dependence for the matched cross sections.

*** The European Physical Society Conference on High Energy Physics (EPS-HEP2021), *** *** 26-30 July $2021 * * *$

*** Online conference, jointly organized by Universität Hamburg and the research center DESY ***

${ }^{*}$ Speaker 


\section{Introduction}

Processes involving the top quark and the Higgs boson are of particular interest for the search of new physics, because they provide direct access to the Yukawa coupling. The associated single top and Higgs production provides a very important test for the Standard Model: although its production rate is smaller at the LHC than the one for $t \bar{t} H$ [1], it provides information not only on the magnitude of the Yukawa coupling, but also on its relative sign to the coupling of the Higgs boson to a $W$ boson. Since it is measured by experiments together with $t \bar{t} H$, accurate theoretical predictions need to be obtained with the same accuracy as the latter [2, 3].

Fixed order calculations at next-to-leading-order (NLO) QCD for single top and Higgs production have been available for some time [4] and, recently, NLO QCD+EW results have been obtained for all combined channels [5]. While NNLO results for such a process are technically out of reach, already some results for approximate NNLO (aNNLO) in the $t$-channel have been reported [6, 7]. They account for contributions coming from soft gluon emission at NNLO. Such emission will lead to logarithmic terms of the form $\alpha_{s}^{n}\left[\log ^{m}(1-\hat{\rho}) /(1-\hat{\rho})\right]_{+}, m \leq 2 n-1$, appearing in the cross section at all orders in $\alpha_{s}$. In the work reported here, we aim to include the effects of such gluon emission at all orders in $\alpha_{s}$ by performing soft gluon resummation at the net-to-leading logarithmic (NLL) accuracy, supplemented by $O\left(\alpha_{s}\right)$ non-logarithmic terms. In order to develop the necessary techniques, we focus first on the calculations for the $s$-channel $t H$ production.

In the following we present results for resummed cross section in the invariant mass threshold, i.e. $\hat{\rho}=Q^{2} / \hat{s} \rightarrow 1$ with $Q^{2}=\left(p_{t}+p_{H}+p_{j}\right)^{2}$, for a three-particle invariant mass kinematic configuration using the Mellin-space approach.

\section{Resummation at invariant mass threshold}

The partonic resummed cross section at next-to-leading-logarithmic (NLL) accuracy in Mellin space can be written as $[8,9]$ :

$$
\tilde{\hat{\sigma}}_{i j}^{(\mathrm{NLL})}=\operatorname{Tr}\left[\mathbf{H}_{i j \rightarrow t H k} \mathbf{S}_{i j \rightarrow t H k}\right] \Delta_{i} \Delta_{j} J_{k},
$$

where $\mathbf{H}_{i j \rightarrow t H k}$ accounts for the hard dynamics and $\mathbf{S}_{i j \rightarrow t H k}$ contains soft-wide angle emission contributions, both of them being matrices in colour space. The soft-collinear emission from the incoming parton $i(j)$ is gathered in the incoming jet function $\Delta_{i}\left(\Delta_{j}\right)$, while the corresponding soft-collinear emission from the outgoing jet $k$ is gathered in the outgoing jet function $J_{k}$. The evolution of the soft function is driven by the soft anomalous dimension matrix, which at NLL accuracy is only needed at one loop $\boldsymbol{\Gamma}^{(1)}$. For simplicity, we work in the colour basis where $\boldsymbol{\Gamma}^{(1)}$ is diagonal,

$$
\Gamma_{R, I J}^{(1)}=\lambda_{I}^{(1)} \delta_{I J}
$$

with $\lambda_{I}^{(1)}$ the eigenvalues of $\boldsymbol{\Gamma}^{(1)}$ and $R$ denoting such basis. The one-loop soft anomalous dimension matrix has been analytically calculated using a suitable $s$-channel colour basis and cross checked against published results [6]. At NLL, one can then write the soft part in this basis as

$$
\mathbf{S}_{R, I J}=\tilde{\mathbf{S}}_{R, I J} \exp \left[\frac{\log (1-2 \lambda)}{2 \pi b_{0}}\left(\left(\lambda_{I}^{(1)}\right)^{*}+\lambda_{J}^{(1)}\right)\right] .
$$


The hard and soft functions can be both expanded perturbatively in powers of $\alpha_{s}: \mathbf{H}_{R}=\mathbf{H}_{R}^{(0)}+$ $\frac{\alpha_{s}}{\pi} \mathbf{H}_{R}^{(1)}+\ldots$ and $\tilde{\mathbf{S}}_{R}=\tilde{\mathbf{S}}_{R}^{(0)}+\frac{\alpha_{s}}{\pi} \tilde{\mathbf{S}}_{R}^{(1)}+\ldots$ To perform resummation at NLL accuracy, only the lowest order terms are needed. The accuracy of the predictions can be improved beyond NLL by including non-logarithmic contributions at $O\left(\alpha_{s}\right)$, i.e. $\mathbf{H}^{(1)}$ and $\tilde{\mathbf{S}}^{(1)}$. Considering the combination of $\mathbf{H}_{R}$ and $\tilde{\mathbf{S}}_{R}$ only up to $O\left(\alpha_{s}\right)$ - referred to as the $\mathbf{C}_{R}^{(1)}$ coefficient - the product of hard and soft matrices can be written as

$$
\mathbf{H}_{R} \tilde{\mathbf{S}}_{R}=\mathbf{H}_{R}^{(0)} \tilde{\mathbf{S}}_{R}^{(0)}\left(\mathbb{1}+\frac{\alpha_{\mathrm{s}}}{\pi} \mathbf{C}_{R}^{(1)}\right)
$$

Taking into account such contributions leads to a cross section at the accuracy which we refer to as "NLLwC". The $\mathbf{C}_{R}^{(1)}$ coefficient gathers, among others, contributions from one-loop virtual corrections and hard-collinear contributions from the incoming and outgoing quark jets. With such a contribution, the differential partonic cross section at the NLLwC accuracy can be expressed as

$$
\begin{aligned}
\frac{d \tilde{\hat{\sigma}}_{i j}^{(\mathrm{NLLwC})}}{d Q^{2}} & \left(N, Q^{2},\left\{m^{2}\right\}, \mu_{\mathrm{F}}^{2}, \mu_{\mathrm{R}}^{2}\right)= \\
& \left(\mathbf{H}_{R, I J} \tilde{\mathbf{S}}_{R, J I}\right)\left(Q^{2},\left\{m^{2}\right\}, \mu_{\mathrm{F}}^{2}, \mu_{\mathrm{R}}^{2}, \delta\right) \\
& \times \Delta_{i}\left(N+1, Q^{2}, \mu_{\mathrm{F}}^{2}, \mu_{\mathrm{R}}^{2}\right) \Delta_{j}\left(N+1, Q^{2}, \mu_{\mathrm{F}}^{2}, \mu_{\mathrm{R}}^{2}\right) \\
& \times J_{k}\left(N+1, \delta, Q^{2}, \mu_{\mathrm{R}}^{2}\right) \exp \left[\frac{\log (1-2 \lambda)}{2 \pi b_{0}}\left(\left(\lambda_{J}^{(1)}\right)^{*}+\lambda_{I}^{(1)}\right)\right] .
\end{aligned}
$$

We treat the final state $b$-quark as massless, and define a final state jet with jet radius $R$ in the small cone approximation. We also consider two different cases concerning the treatment of the jet mass, either treating it as massless $\left(p_{j}^{2}=0\right)$ or allowing it to be massive $\left(p_{j}^{2} \neq 0\right)$ [9]. The exact results for the $\mathbf{H}_{R}^{(0)}, \tilde{\mathbf{S}}_{R}^{(0)}, \mathbf{C}_{R}^{(1)}$, as well as the incoming and outgoing jet functions can be found in [10].

The resummation-improved cross sections NLO+NLLwC are then obtained through the matching procedure, i.e. the combination of fixed-order calculations and resummed results,

$$
\begin{aligned}
\frac{d \sigma_{h_{1} h_{2}}^{(\mathrm{NLO}+\mathrm{NLLwC})}}{d Q^{2}}\left(Q^{2},\left\{m^{2}\right\}, \mu_{\mathrm{F}}^{2}, \mu_{\mathrm{R}}^{2}\right) & =\frac{d \sigma_{h_{1} h_{2}}^{(\mathrm{NLO})}}{d Q^{2}}\left(Q^{2},\left\{m^{2}\right\}, \mu_{\mathrm{F}}^{2}, \mu_{\mathrm{R}}^{2}\right) \\
& +\frac{d \sigma_{h_{1} h_{2}}^{(\text {(res-exp })}}{d Q^{2}}\left(Q^{2},\left\{m^{2}\right\}, \mu_{\mathrm{F}}^{2}, \mu_{\mathrm{R}}^{2}\right)
\end{aligned}
$$

with

$$
\begin{aligned}
& \frac{d \sigma_{h_{1} h_{2}}^{(\mathrm{res}-\mathrm{exp})}}{d Q^{2}}\left(Q^{2},\left\{m^{2}\right\}, \mu_{\mathrm{F}}^{2}, \mu_{\mathrm{R}}^{2}\right)=\sum_{i, j=\left\{q, \bar{q}^{\prime}\right\}} \int_{\mathrm{C}} \frac{d N}{2 \pi i} \rho^{-N} f_{i / h_{1}}^{(N+1)}\left(\mu_{\mathrm{F}}^{2}\right) f_{j / h_{2}}^{(N+1)}\left(\mu_{\mathrm{F}}^{2}\right) \\
& \times\left[\frac{d \tilde{\hat{\sigma}}_{q \bar{q}^{\prime}}^{(\mathrm{NLLwC})}}{d Q^{2}}\left(N, Q^{2},\left\{m^{2}\right\}, \mu_{\mathrm{F}}^{2}, \mu_{\mathrm{R}}^{2}\right)-\left.\frac{d \tilde{\hat{\sigma}}_{q \bar{q}^{\prime}}^{(\mathrm{NLLwC})}}{d Q^{2}}\left(N, Q^{2},\left\{m^{2}\right\}, \mu_{\mathrm{F}}^{2}, \mu_{\mathrm{R}}^{2}\right)\right|_{(\mathrm{NLO})}\right],
\end{aligned}
$$

where the last term in the brackets corresponds to the expansion of the resummed cross section truncated at NLO. This subtraction is needed to avoid double counting in the matching procedure. 


\section{Numerical results}

We next present the numerical results for soft gluon resummation at NLO+NLLwC accuracy for the $s$-channel of the associated single top and Higgs production at $\sqrt{S}=13 \mathrm{TeV}$, which were obtained using $m_{t}=173 \mathrm{GeV}, m_{H}=125 \mathrm{GeV}$ and PDF4LHC15 PDF sets [11-16]. NLO cross sections and the one-loop virtual corrections - needed to implement the $\mathbf{C}^{(1)}$ coefficient - have been numerically obtained from aMC@NLO code [17]. The results have been computed taking the the renormalization and factorization scales equal, i.e. $\mu_{R}=\mu_{F}=\mu$.

In Figure 1 we present invariant mass differential distributions for both jet treatments, showing the NLO with the quark-gluon channel subtracted and the expanded resummed result NLLwC $\left.\right|_{\text {NLO }}$. One can see that in general the results are close to each other, although for most of the range of the spectra there is a slight overestimation by the NLLwC $\left.\right|_{N L O}$ result. Our calculations also show the importance of the power-subleading terms in $N$, partly contributing to the numerical differences beyond $O\left(\alpha_{s}\right)$ for the two different jet treatments [10].
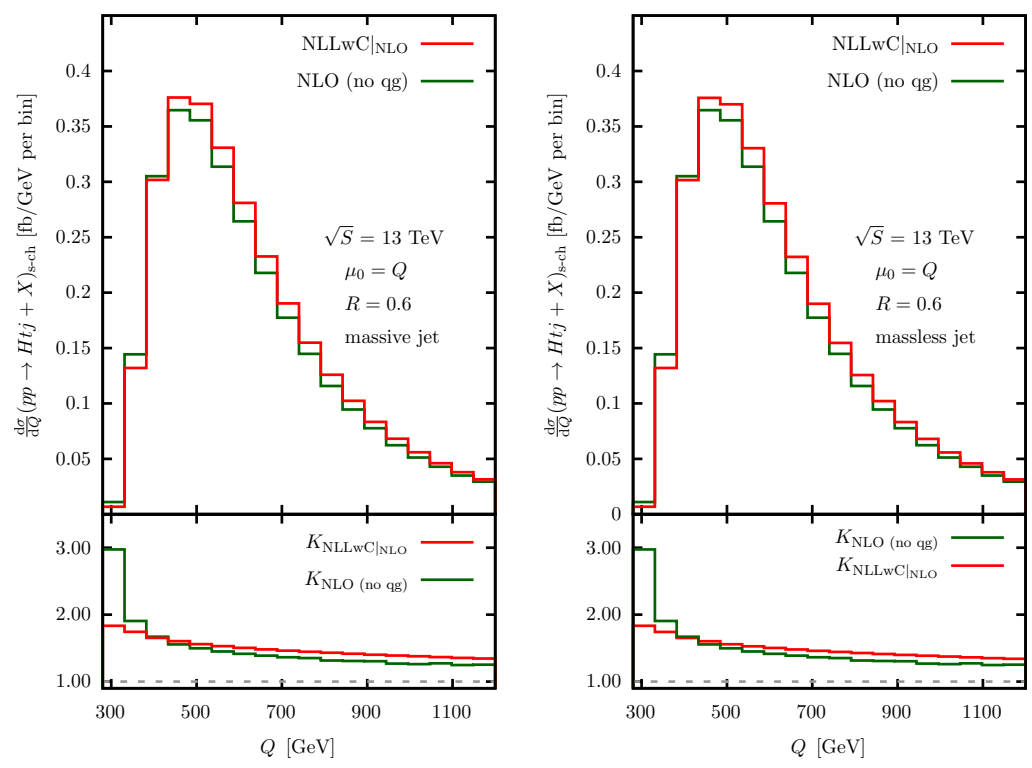

Figure 1: Differential distributions in $Q$ for massive (left plot) and massless (right plot) jets for $R=0.6$ : (top) absolute values of the NLO(no qg), NLLwC|NLO distributions and (bottom) ratios of NLLwC|NLO and $\mathrm{NLO}$ (no qg) results to the $\mathrm{LO}$ predictions.

In Figure 2 we show the scale dependence of the total cross sections calculated with different theoretical precision. From the big jump between LO and the rest of the results, one can notice the importance of including higher order contributions, with NLO above $35 \%$ of the LO result for all the range of $\mu$ considered. In line with the results shown in Figure 1, the overestimation is carried over to the integrated results. The resummed results expanded up to NLO for the two different jet treatments are in a very good agreement.

In Figure 3 we consider NLO+NLLwC and NLO total cross sections for several central scale choices $\mu=\mu_{0}$. Additionally, we also study the dependence of the results on the scale $\mu$. One can see that in comparison with the fixed order NLO predictions, the scale dependence is reduced 


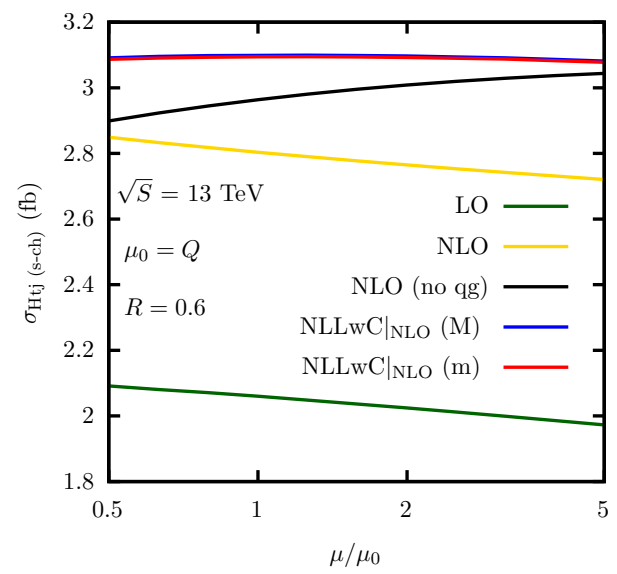

Figure 2: Scale dependence of the total $p p \rightarrow H t j$ cross section for $R=0.6$ at the LO, NLO (with and without the $q g$ channel) and NLLwC $\left.\right|_{\text {NLO }}$ accuracy with massive (M) and massless (m) final state jet.

for the individual matched results. Furthermore, the NLO+NLLwC results calculated for different $\mu_{0}$ values are less spread than the corresponding NLO results. The spread for matched results stays smaller even after taking into account differences having their origins in how the outgoing jet is treated. This demonstrates the importance of soft gluon emission effects for the theoretical predictions for the single top and Higgs production at the LHC.
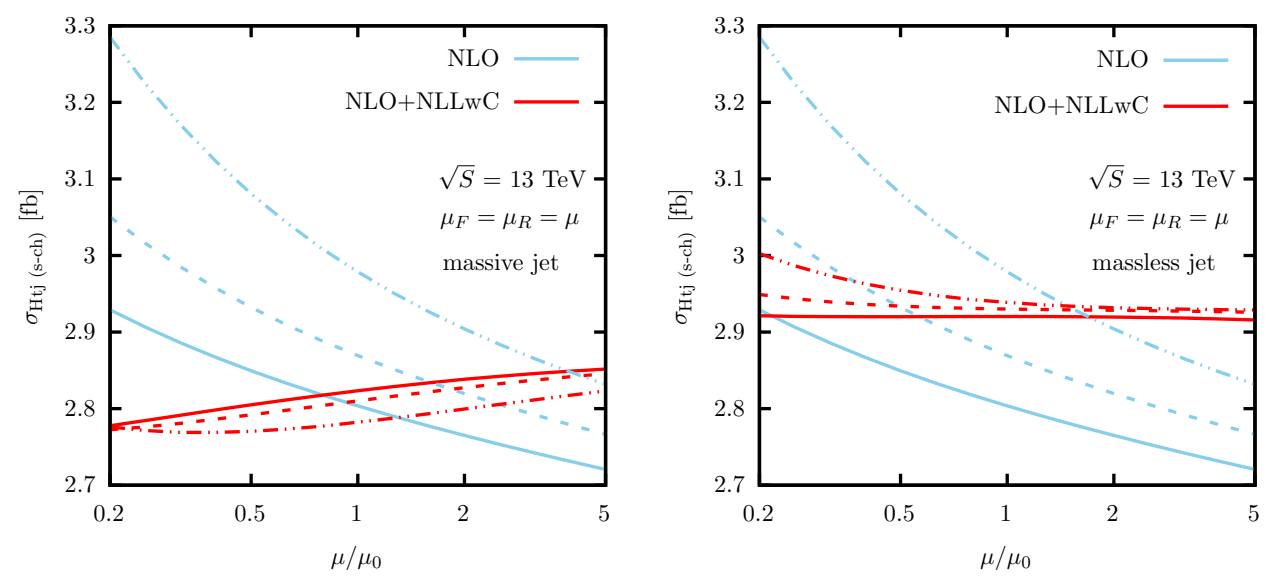

Figure 3: Scale dependence of the total $p p \rightarrow H t j$ cross section for $R=0.6$ at the NLO and NLO+NLLwC accuracy for massive (left plot) and massless (right plot) jet treatment. Results are shown for three different central scale choices $\mu_{0}=Q$ (solid lines), $\mu_{0}=H_{T} / 2$ (dashed lines), and $\mu_{0}=H_{T} / 6$ (dashed-dotted lines).

\section{Acknowledgments}

This work has been supported in part by the Deutsche Forschungsgemeinschaft (DFG) grant KU3103/2. V.T. acknowledges funding from the European Union's Horizon 2020 research and inno- 
vation programme as part of the Marie Skłodowska-Curie Innovative Training Network MCnetITN3 (grant agreement no. 722104), while L.M.V. acknowledges support from the DFG Research Training Group "GRK 2149: Strong and Weak Interactions - from Hadrons to Dark Matter". L.M.V. would also like to thank the organizers of the conference for the possibility to present this talk.

\section{References}

[1] F. Maltoni, K. Paul, T. Stelzer and S. Willenbrock, Phys. Rev. D 64 (2001), 094023 [arXiv:hepph/0106293 [hep-ph]].

[2] A. Kulesza, L. Motyka, D. Schwartländer, T. Stebel and V. Theeuwes, Eur. Phys. J. C 80 (2020) no.5, 428 [arXiv:2001.03031 [hep-ph]].

[3] A. Broggio, A. Ferroglia, R. Frederix, D. Pagani, B. D. Pecjak and I. Tsinikos, JHEP 08 (2019), 039 [arXiv:1907.04343 [hep-ph]].

[4] F. Demartin, F. Maltoni, K. Mawatari and M. Zaro, Eur. Phys. J. C 75 (2015) no.6, 267 [arXiv:1504.00611 [hep-ph]].

[5] D. Pagani, I. Tsinikos and E. Vryonidou, JHEP 08 (2020), 082 [arXiv:2006.10086 [hep-ph]].

[6] M. Forslund and N. Kidonakis, Phys. Rev. D 102 (2020) no.3, 034006 [arXiv:2003.09021 [hep-ph]].

[7] M. Forslund and N. Kidonakis, [arXiv:2103.01228 [hep-ph]].

[8] H. Contopanagos, E. Laenen and G. F. Sterman, Nucl. Phys. B 484 (1997) 303 [hep$\mathrm{ph} / 9604313]$.

[9] N. Kidonakis, G. Oderda and G. Sterman, Nucl. Phys. B 531, 365 (1998). [arXiv:hep$\mathrm{ph} / 9803241]$.

[10] A. Kulesza, L. M. Valero and V. Theeuwes, [arXiv:2109.15039 [hep-ph]].

[11] J. Butterworth et al., J. Phys. G 43 (2016) 023001 [arXiv:1510.03865 [hep-ph]].

[12] S. Dulat et al., Phys. Rev. D 93 (2016) no.3, 033006 [arXiv:1506.07443 [hep-ph]].

[13] L. A. Harland-Lang, A. D. Martin, P. Motylinski and R. S. Thorne, Eur. Phys. J. C 75 (2015) no.5, 204 [arXiv:1412.3989 [hep-ph]].

[14] R. D. Ball et al. [NNPDF Collaboration], JHEP 1504 (2015) 040 [arXiv:1410.8849 [hep-ph]].

[15] J. Gao and P. Nadolsky, JHEP 1407 (2014) 035 [arXiv:1401.0013 [hep-ph]].

[16] S. Carrazza, S. Forte, Z. Kassabov, J. I. Latorre and J. Rojo, Eur. Phys. J. C 75 (2015) no.8, 369 [arXiv:1505.06736 [hep-ph]].

[17] J. Alwall et al., JHEP 1407 (2014) 079 [arXiv:1405.0301 [hep-ph]]. 